\title{
Amplitude death in nonlinear oscillators with mixed time-delayed coupling
}

\author{
Wei Zou,,${ }^{1,2,3, *}$ D. V. Senthilkumar, ${ }^{3}$ Yang Tang, ${ }^{2,3}$ Ye Wu, ${ }^{3,4}$ Jianquan Lu, ${ }^{5}$ and Jürgen Kurths $s^{2,3,6}$ \\ ${ }^{1}$ School of Mathematics and Statistics, Huazhong University of Science and Technology, Wuhan 430074, China \\ ${ }^{2}$ Institute of Physics, Humboldt University Berlin, Berlin D-12489, Germany \\ ${ }^{3}$ Potsdam Institute for Climate Impact Research, Telegraphenberg, Potsdam D-14415, Germany \\ ${ }^{4}$ School of Science, Beijing University of Posts and Telecommunications, Beijing 100876, China \\ ${ }^{5}$ Department of Mathematics, Southeast University, Nanjing 210096, China \\ ${ }^{6}$ Institute for Complex Systems and Mathematical Biology, University of Aberdeen, Aberdeen AB24 3FX, United Kingdom
}

(Received 25 February 2013; published 25 September 2013)

\begin{abstract}
Amplitude death (AD) is an emergent phenomenon whereby two or more autonomously oscillating systems completely lose their oscillations due to coupling. In this work, we study AD in nonlinear oscillators with mixed time-delayed coupling, which is a combination of instantaneous and time-delayed couplings. We find that the mixed time-delayed coupling favors the onset of $\mathrm{AD}$ for a larger set of parameters than in the limiting cases of purely instantaneous or completely time-delayed coupling. Coupled identical oscillators experience AD under instantaneous coupling mixed with a small proportion of time-delayed coupling. Our work gives a deeper understanding of delay-induced AD in coupled nonlinear oscillators.
\end{abstract}

DOI: 10.1103/PhysRevE.88.032916

PACS number(s): 05.45.Xt, 87.10.-e

\section{INTRODUCTION}

Collective behavior in a large ensemble of real-life systems may be qualitatively reproduced and investigated in a simple model of coupled nonlinear oscillators [1-5]. Exploring the dynamics of coupled nonlinear oscillators has become an active field of research over the past several decades, with applications ranging from physics to chemistry, biology, and even social sciences. Depending on the intrinsic properties of individual oscillators and the external interactions between them, coupled nonlinear oscillators can give rise to a host of collective phenomena such as synchronization [6,7], chimera states [8,9], phase flips [10,11], etc.

Amplitude death (AD) refers to a dynamical phenomenon where the oscillations of coupled oscillatory systems are completely suppressed as a consequence of their interaction $[3,5,11]$. The phenomenon of AD has been observed in the different contexts of chemical reactions [12-14], biological oscillators [15-18], laser systems [19-21], and relativistic magnetrons [22]. AD is of particular importance in controlling (or even maintaining) the oscillatory dynamics of realistic systems, and therefore understanding the onset conditions of $\mathrm{AD}$ is crucial. In general AD occurs in instantaneously coupled oscillators only if their intrinsic frequencies have a fairly broad distribution [23-25]. However, in 1998, Reddy, Sen, and Johnston reported that inclusion of an appropriate time delay in the coupling induces AD even in coupled identical oscillators without any frequency mismatch [26]. This phenomenon was also named "death by delay" by Strogatz [27], and subsequently has been experimentally realized in nonlinear electronic circuits [28]. Recent investigations have revealed that AD can also occur in coupled identical oscillators under other coupling schemes such as dynamical coupling [29-31], conjugate coupling [32-34], nonlinear coupling [35], indirect coupling [36,37], and mean-field diffusion [38], etc.

*zouwei2010@mail.hust.edu.cn
In physical or biological systems, time delay typically arises from a finite propagation speed or processing time of signals. Thus it is important and reasonable to take time delay into account when analyzing the dynamics of coupled systems. In this connection, investigations of delay-induced AD in coupled nonlinear oscillators have been an active area of research for over a decade. For example, it has been proposed that different kinds of delayed interaction among coupled nonlinear oscillators successfully generate AD, such as distributed time delays [39], partial time delays [40], integrative time delays [41], a time-varying delay [42], and multiple time delays $[43,44]$. Some works addressed the effects of the nature of the coupling on delay-induced $\mathrm{AD}$ such asthe coupling gradient [45], the coupling phase [46,47], and the coupling asymmetry [48]. The study of delay-induced AD was extended from coupled limit-cycle systems [49] to coupled chaotic oscillators [50] and coupled time-delayed oscillators [51,52]. The phenomenon of delay-induced AD has been found in coupled oscillator networks with distinct topologies, e.g., an all-to-all network [53], a ring network [54], a one-way ring [55], and complex networks [56,57].

Instantaneous and time-delayed coupling schemes are the two limiting cases of a more general situation, where the coupling signal often contains combinations of both instantaneous and time-delayed contributions. Theoretical considerations of both the limiting cases may represent an exceptional nature of interactions in real-world systems, while the presence of both instantaneous and time-delayed interactions with a certain distribution would be a more natural one. In this paper, we study the emergence of AD in nonlinear oscillators with mixed time-delayed coupling, where the coupling is constructed with a proportioned mixture of instantaneous and time-delayed couplings. Such mixed time-delayed coupling in fact acts as a bridge linking the purely instantaneous coupling and the fully time-delayed coupling, and is more realistic in many applications. Because a certain portion of the timedelayed coupling is replaced by instantaneous interaction, the contribution of the time-delayed effect is weakened. One may intuitively speculate that mixed time-delayed coupling is 
detrimental to the persistence of delay-induced AD in identical nonlinear oscillators. In contrast, our results in this paper elucidate that on increasing the proportion of the instantaneous coupling, $\mathrm{AD}$ occurs for a larger set of parameters for the mixed time-delayed coupling rather than the case of fully time-delayed coupling. The instantaneous coupling mixed with a small proportion of time-delayed coupling is indeed capable of producing AD in coupled identical oscillators.

The rest of this paper is organized as follows. Section II focuses on the analysis of $\mathrm{AD}$ in two coupled limit-cycle oscillators with mixed time-delayed coupling. Sections III and IV generalize the results to networked limit-cycle oscillators and coupled chaotic oscillators, respectively. Finally, conclusions and discussion are provided in Section V.

\section{TWO COUPLED LIMIT-CYCLE OSCILLATORS}

Let us start with the following system of two Stuart-Landau limit-cycle oscillators with mixed time-delayed coupling:

$$
\begin{aligned}
\dot{z}_{1}= & \left(1+i w_{1}-\left|z_{1}\right|^{2}\right) z_{1}+(1-\alpha) K\left(z_{2}-z_{1}\right) \\
& +\alpha K\left[z_{2}(t-\tau)-z_{1}(t)\right], \\
\dot{z}_{2}= & \left(1+i w_{2}-\left|z_{2}\right|^{2}\right) z_{2}+(1-\alpha) K\left(z_{1}-z_{2}\right) \\
& +\alpha K\left[z_{1}(t-\tau)-z_{2}(t)\right],
\end{aligned}
$$

where $z_{1}$ and $z_{2}$ are complex variables, $i=\sqrt{-1}$ is the imaginary unit, $w_{1}$ and $w_{2}$ are the intrinsic frequencies of two uncoupled limit-cycle oscillators, $K \geqslant 0$ is the strength of the diffusive coupling, $\tau$ is the amount of time delay, and $\alpha(0 \leqslant$ $\alpha \leqslant 1$ ) determines the proportion of the instantaneous and time-delayed interactions in the coupling. In the case of $\alpha=0$, the coupling is purely instantaneous; and if $\alpha=1$, the coupling is completely time delayed. These two extreme situations have already been well analyzed by Aronson et al. [23] and Reddy et al. [26], respectively. For intermediate values of $\alpha$ $(0<\alpha<1)$, both the instantaneous and time-delayed effects contribute to the coupling. This work is aimed to investigate the impact of the parameter $\alpha$ on the onset of AD in coupled nonlinear oscillators.

Stuart-Landau oscillator is a prototypical limit-cycle system experiencing a supercritical Hopf bifurcation. Employing this model can reveal universal features of many practical systems, where the oscillation arises through a Hopf bifurcation. In fact, systems of coupled Stuart-Landau oscillators have been widely used for studying various dynamical behaviors. In particular, it has been used as an ideal paradigmatic model for theoretically exploring mechanisms of AD in coupled nonlinear systems for decades. Without coupling in Eq. (1), i.e., $K=0$, the two uncoupled Stuart-Landau oscillators exhibit stable limitcycle motions with $z_{1}=e^{i w_{1} t}$ and $z_{2}=e^{i w_{2} t}$, and possess the same unstable steady state $z_{1}=z_{2}=0$. When the coupling is turned on, i.e., $K>0$, the stability of unstable origin is changed for appropriate $K$, while the two stable limit cycles collapse into the origin, signaling the onset of the AD phenomenon. This scenario of stabilization of unstable fixed points in coupled Stuart-Landau limit-cycle oscillators by time-delayed coupling is dynamically different from the stabilization of inherently unstable periodic orbits in coupled nonlinear oscillators by delayed feedback as studied in [58].
To detect the occurrence of $\mathrm{AD}$ in the coupled system Eq. (1), we linearize this system around the origin $z_{1}=z_{2}=0$. By assuming all the linear perturbations to be proportional to $e^{\lambda t}$, the corresponding characteristic equation is obtained as

$$
\begin{aligned}
& \left(1+i w_{1}-K-\lambda\right)\left(1+i w_{2}-K-\lambda\right) \\
& \quad=\left[(1-\alpha) K+\alpha K e^{-\lambda \tau}\right]^{2},
\end{aligned}
$$

where $\lambda$ is the complex eigenvalue. This is a transcendental equation, which has an infinite number of roots but only a finite number of roots with positive real part. If all the real parts are negative, then the origin is stabilized, leading to the phenomenon of AD.

The stability of $\mathrm{AD}$ for $\alpha=0$ has been studied in great detail in [23], leading to the conclusion that both the coupling strength and the frequency mismatch need to be sufficiently large in order to induce AD. The necessary and sufficient conditions have been explicitly derived as $1<K<\left(1+\Delta^{2} / 4\right) / 2$ and $\Delta>2$, where $\Delta=\left|w_{1}-w_{2}\right|$ quantifies the frequency mismatch of the two oscillators. Thus, AD is impossible for diffusively coupled identical oscillators without time delay. Figure 1(a) shows the AD region in the parameter space of $(K, \Delta)$. The $\mathrm{AD}$ region is marked by the shaded area.

Reddy et al. confined their studies to the case of fully time-delayed coupling for $\alpha=1$ [26]. They found that in the presence of time delay the $\mathrm{AD}$ region on the $(K, \Delta)$ plane is distorted, and can even be extended down to the $\Delta=0$ axis for a certain range of values of the time delay $\tau$. Therefore, $\mathrm{AD}$ is achieved for coupled identical oscillators if the coupling is time delayed. To illustrate the effect of mixed time-delayed coupling, here we intentionally fix the time delay at $\tau=0.2$, and the frequencies as $w_{1}=10+\frac{\Delta}{2}$ and $w_{2}=10-\frac{\Delta}{2}$. For this value of $\tau$, the $\mathrm{AD}$ region is just deformed but far from $\Delta=0$ for the fully time-delayed coupling case [comparing Figs. 1(a) and 1(b)]. Surprisingly, we find that the AD region extends downwards to the $\Delta=0$ axis when a portion of the time-delayed coupling is replaced with the instantaneous one.

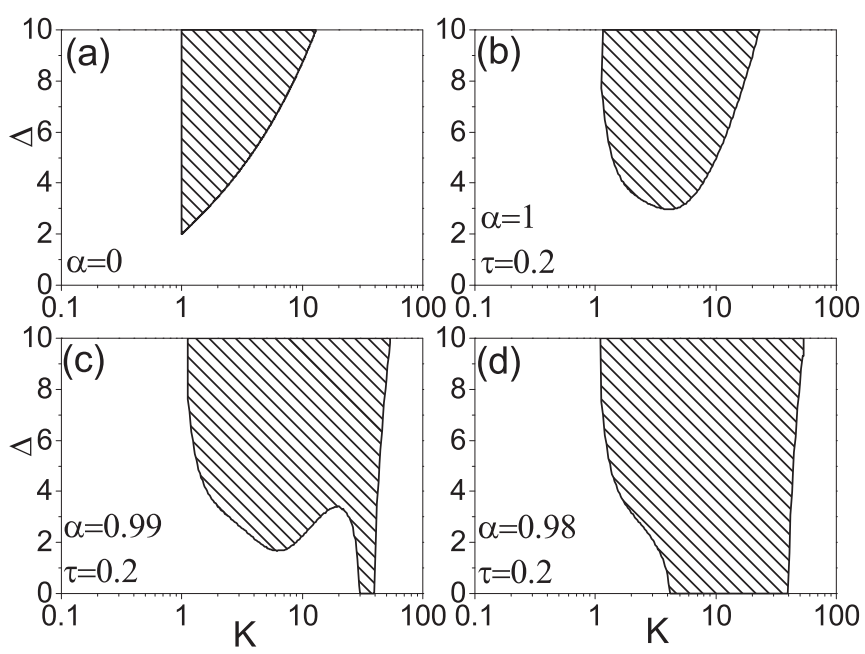

FIG. 1. The stability regions of AD in two coupled nonidentical oscillators described by Eq. (1) for (a) the purely instantaneous coupling with $\alpha=0$, (b) the fully time-delayed coupling with $\alpha=1.0$, and the mixed time-delayed coupling with (c) $\alpha=0.99$ and (d) $\alpha=0.98$, respectively. $w_{1}=10+\Delta / 2$ and $w_{2}=10-\Delta / 2$. $\tau=0.2$ is fixed. 
For instance, see the AD regions shown in Figs. 1(c) and 1(d) for two different values of the parameter $\alpha=0.99$ and 0.98. Clearly, the presence of a small fraction of instantaneous coupling along with the time-delayed coupling facilitates the onset of AD. Such an effect of mixed time-delayed coupling is more pronounced as the value of $\alpha$ is gradually decreased.

To examine the influence of the parameter $\alpha$ on the conditions of the onset of $\mathrm{AD}$ in coupled identical oscillators, we take a detailed look at the $\mathrm{AD}$ region in the parameter space of $(\tau, K)$ for $\Delta=0$. Setting $w_{1}=w_{2}=w$, the characteristic equation (2) is simplified to the following two equations:

$$
\lambda=1+i w-\alpha K+\alpha K e^{-\lambda \tau}
$$

and

$$
\lambda=1+i w-(2-\alpha) K-\alpha K e^{-\lambda \tau} .
$$

The phenomenon of AD occurs if and only if all the roots of both Eqs. (3) and (4) have negative real parts.

Using the complex Lambert function $W$, which is the inverse function of $g(\Lambda)=\Lambda e^{\Lambda}$ for complex $\Lambda$ [59,60], the roots for Eqs. (3) and (4) can be obtained analytically as

$$
\lambda=\frac{1}{\tau} W\left(\tau \alpha K e^{-(1+i w-\alpha K) \tau}\right)+1+i w-\alpha K
$$

and

$$
\lambda=\frac{1}{\tau} W\left(-\tau \alpha K e^{-(1+i w-2 K+\alpha K) \tau}\right)+1+i w-(2-\alpha) K,
$$

respectively. Further, based on a simple observation of Eqs. (3) and (4), we notice that the stability of the origin could be switched only if an eigenvalue $\lambda$ crosses the imaginary axis as the parameters are changed. Thus at the critical point, the characteristic root is purely imaginary, i.e., the characteristic root can be expressed as $\lambda=i \lambda_{I}$. Substituting into Eqs. (3) and (4), and after some straightforward mathematical manipulations, we derive the following set of four critical curves bounding the $\mathrm{AD}$ region:

$$
\begin{aligned}
\tau_{a} & =\frac{2 m \pi+\cos ^{-1}\left(1-\frac{1}{\alpha K}\right)}{w-\sqrt{2 \alpha K-1}}, \\
\tau_{b} & =\frac{2(m+1) \pi-\cos ^{-1}\left(1-\frac{1}{\alpha K}\right)}{w+\sqrt{2 \alpha K-1}}, \\
\tau_{c} & =\frac{2(m+1) \pi-\cos ^{-1}\left[\frac{1-(2-\alpha) K}{\alpha K}\right]}{w-\sqrt{(2 K-1)(1-2 K+2 \alpha K)}}, \\
\tau_{d} & =\frac{2 m \pi+\cos ^{-1}\left[\frac{1-(2-\alpha) K}{\alpha K}\right]}{w+\sqrt{(2 K-1)(1-2 K+2 \alpha K)}},
\end{aligned}
$$

where $m=0,2, \ldots, \infty$, denoting the possible number of $\mathrm{AD}$ islands. $\tau_{a}$ and $\tau_{b}$ are the critical curves deduced from Eq. (3), $\tau_{c}$ and $\tau_{d}$ from Eq. (4). With a procedure similar to that in $[26,39]$, the enclosed AD region is finally identified by further looking at the behavior of the quantity $\operatorname{Re}\left(\frac{\partial \lambda}{\partial \tau}\right)$ calculated from Eqs. (3) and (4) by implicit differentiation on the four critical curves $\tau_{a}, \tau_{b}, \tau_{c}$, and $\tau_{d}$, respectively. For $\alpha=1$, it has already been shown that only one AD island can be formed in the parameter space of $(\tau, K)$ for $w=10$, and more than one island may appear for high values of $w[26,53,54]$. Without loss of generality, we consider $w \leqslant 10$ for coupled identical
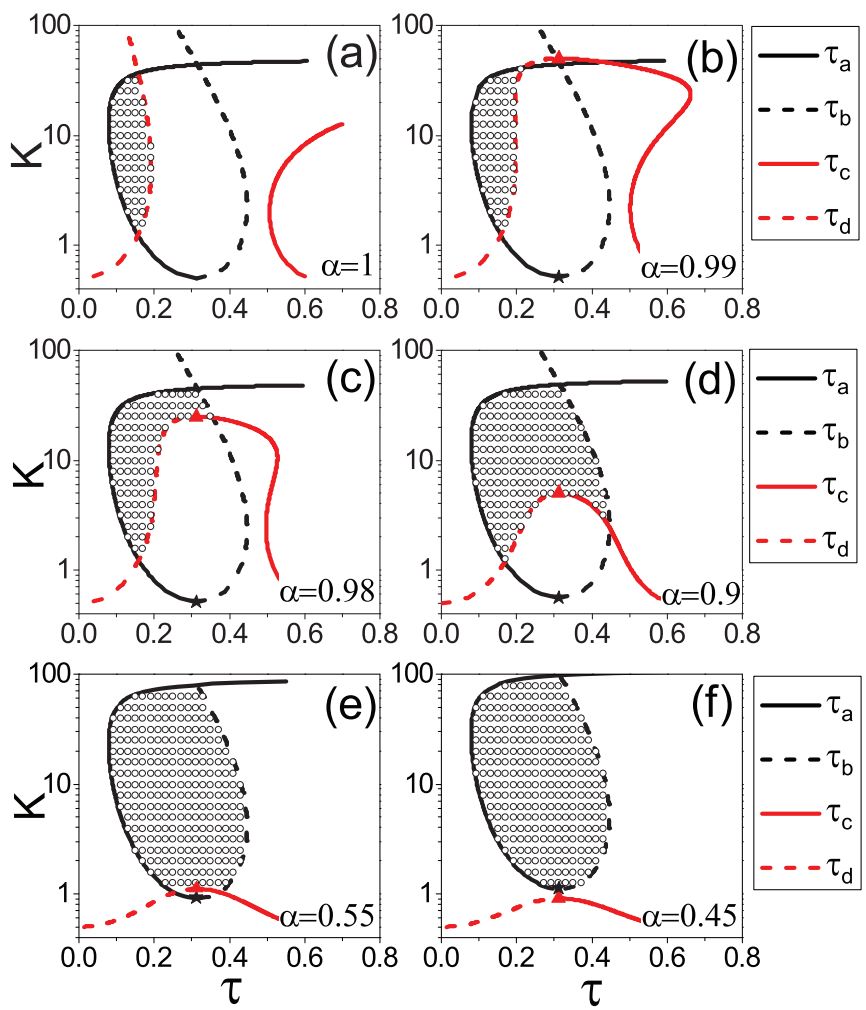

FIG. 2. (Color online) The AD islands of the two coupled identical oscillators of Eq. (1) on the $(\tau, K)$ space for different values of the mixing parameter $\alpha . \alpha=1,0.99,0.98,0.9,0.55$, and 0.45 for (a)-(f), respectively. The AD islands are surrounded by the critical curves $\tau_{a}, \tau_{b}, \tau_{c}$, and $\tau_{d}$ in Eq. (7), which are indicated by the different colors and styles of lines. The black star represents the minimal point of intersection of $\tau_{a}$ and $\tau_{b}:\left(\tau_{a}=\tau_{b}=\pi / w, K=1 / 2 \alpha\right)$; and the red triangle is the maximal point of intersection of $\tau_{c}$ and $\tau_{d}:\left[\tau_{c}=\tau_{d}=\pi / w, K=1 / 2(1-\alpha)\right]$. The open circles are the numerical results, which are in good agreement with the theoretical predictions. $w_{1}=w_{2}=w=10$ is fixed.

oscillators, and $m=0$ in Eq. (7). It is to be noted that for large values of $w>10$, where multiple $\mathrm{AD}$ islands are possible, the effects of $\alpha$ on $\mathrm{AD}$ islands are quite similar to the case of $w=10$ as will be discussed in the following.

The analytical critical curves (7) are depicted in Fig. 2 for $w=10$ and for different values of $\alpha$. The open circles represent the numerical results obtained by numerical integration of the original coupled system Eq. (1), where the AD phenomenon is observed. The $\mathrm{AD}$ regions on the $(K, \tau)$ plane are well bounded by the critical curves in Eq. (7). These $\mathrm{AD}$ regions are also labeled as $\mathrm{AD}$ islands. For the purely time-delayed coupling case with $\alpha=1$, only two critical curves $\tau_{a}$ and $\tau_{c}$ are involved in enclosing the AD island, which retrieves the result of Reddy et al. in [26]. If the time-delayed coupling is mixed with a portion of the instantaneous one, i.e., when $0<\alpha<1, \tau_{b}$ and $\tau_{d}$ may also contribute to the $\mathrm{AD}$ island boundaries, as shown in Figs. 2(b)-2(e) for $\alpha=0.99$, $0.98,0.9,0.55$, and 0.45 , respectively. From these figures, it is clear that the spread of the $\mathrm{AD}$ island increases to a larger set of parameters in the $(\tau, K)$ space for decreasing $\alpha$ in the range of $1>\alpha>0.5$, thereby increasing the proportion of instantaneous coupling. With further decrease in $\alpha(0<\alpha<0.5)$, 
the $\mathrm{AD}$ island expands along the direction of coupling strength with a specific scaling $\frac{1}{\alpha}$. The AD boundaries are then given by only $\tau_{a}$ and $\tau_{b}$, as illustrated in Fig. 2(f) for $\alpha=0.45$.

The scaling $\frac{1}{\alpha}$ of the expansion of the AD island along the $K$ direction for $0<\alpha<0.5$ can be explained as follows. From the forms of $\tau_{a}$ and $\tau_{b}$ in Eq. (7), we can find that all the terms containing the coupling strength $K$ are combined with the parameter $\alpha$. The stable region of Eq. (3) enclosed by these two critical curves will be enlarged exactly $\frac{1}{\alpha}$ times along the axis of the coupling strength compared with that in the case of $\alpha=1$. With decreasing value of $\alpha, \tau_{a}$ and $\tau_{b}$ lift up, while $\tau_{c}$ and $\tau_{d}$ move down. It is not difficult to obtain the minimal point of intersection of $\tau_{a}$ and $\tau_{b}$ as $\left(\tau_{a}=\tau_{b}=\frac{\pi}{w}, K=\frac{1}{2 \alpha}\right)$ (marked by the black stars in Fig. 2) and the maximal point of intersection of $\tau_{c}$ and $\tau_{d}$ as $\left(\tau_{c}=\tau_{d}=\frac{\pi}{w}, K=\frac{1}{2(1-\alpha)}\right)$ (marked by the red triangles in Fig. 2). Thus if $\frac{1}{2(1-\alpha)}<\frac{1}{2 \alpha}$, i.e., $\alpha<0.5$, the AD island is bounded only by $\tau_{a}$ and $\tau_{b}$, which accounts for the expanding nature of the AD island as observed in Fig. 2(f).

From the expressions of the critical curves in Eq. (7), one can see that the $\mathrm{AD}$ island in the $(\tau, K)$ space depends on the intrinsic frequency $w$. In our studies, the same effect of the mixed time-delayed coupling on the AD island as shown in Fig. 2 is found to be generically valid for other values of $w$. For each fixed value of $0<\alpha \leqslant 1$, it is also observed that the $\mathrm{AD}$ island monotonically decreases with decreasing value of $w$ and completely vanishes below a critical threshold $w_{\min }(\alpha)$. As illustrative examples, Figs. 3(a) and 3(b) display the AD islands with four different values of $w$ for $\alpha=0.9$ and $\alpha=0.45$, respectively.

For the case of completely time-delayed coupling, the critical threshold of frequency is $w_{\min }(\alpha=1) \approx 4.812[53,61]$. If the frequency $w$ is below this value, $\mathrm{AD}$ never occurs for any combinations of $\tau$ and $K$ in this case. Figure 3 clearly shows that the $\mathrm{AD}$ island exists in the parameter space of $(\tau, K)$ even as $w<4.812$ for the mixed time-delayed coupling case with $\alpha=0.9$ and $\alpha=0.45$. Thus we can directly conjecture that $w_{\min }(\alpha=0.9)<w_{\min }(\alpha=1)$, and hence $w_{\min }(\alpha=0.45)<$ $w_{\min }(\alpha=1)$ holds, which implies that the presence of the

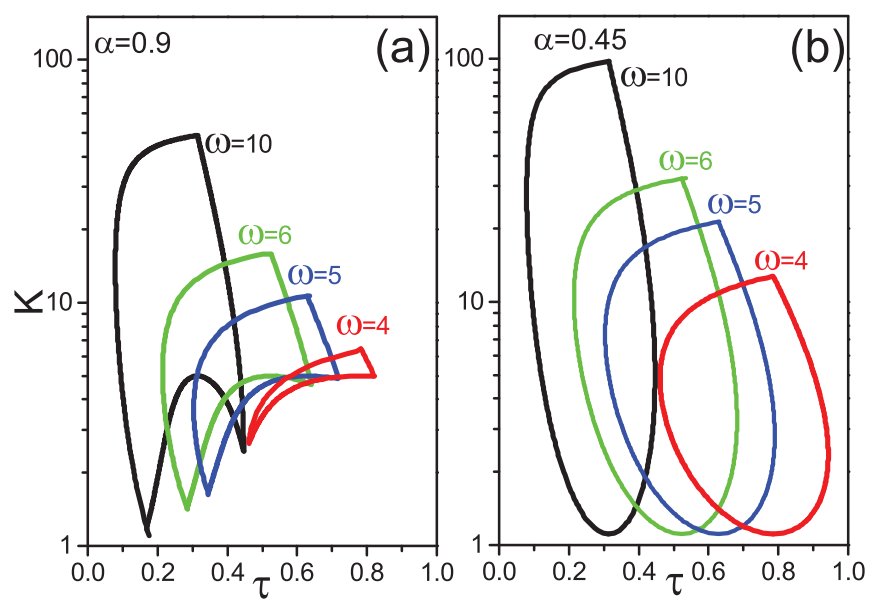

FIG. 3. (Color online) The AD island as a function of the intrinsic frequency $w$ for (a) $\alpha=0.9$ and (b) $\alpha=0.45$. The size of the $\mathrm{AD}$ island monotonically decreases as $w$ decreases. The $\mathrm{AD}$ island disappears if $w$ is below a certain critical value $w_{\min }$.

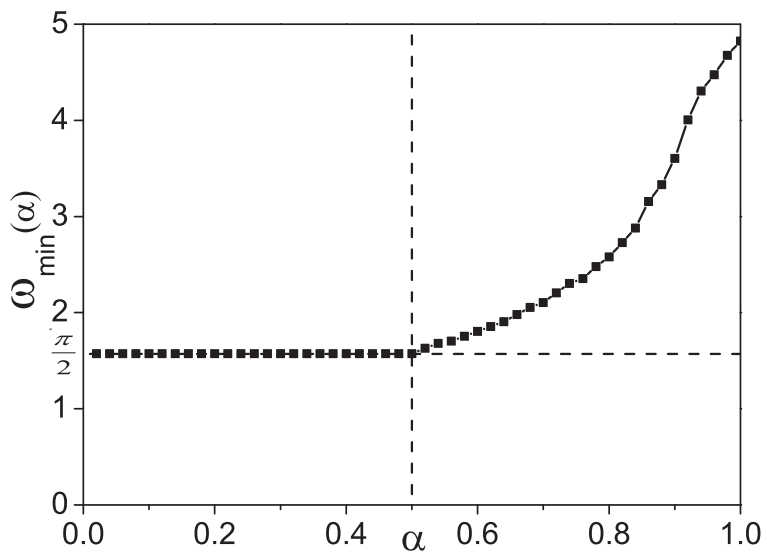

FIG. 4. The critical frequency $w_{\min }(\alpha)$ vs the mixing parameter $\alpha . w_{\min }(\alpha)$ decreases as the mixing parameter $\alpha$ decreases from 1 to 0.5 , and retains at a constant value $\pi / 2$ for $0<\alpha \leqslant 0.5$.

instantaneous coupling along with the time-delayed coupling facilitates the onset of AD even for a small value of the intrinsic frequency $w$.

The critical threshold $w_{\min }(\alpha)$ is numerically calculated by decreasing the value of $w$ at a fixed $\alpha$ until the $\mathrm{AD}$ island disappears. The results are shown Fig. 4, from which we find that $w_{\min }(\alpha)$ exhibits two distinctly different behaviors: (i) if the time-delayed interaction is more than the instantaneous part, i.e., $0.5<\alpha<1, w_{\min }(\alpha)$ monotonically decreases with decreasing $\alpha$ from 1, and (ii) when the proportion of the instantaneous contribution dominates the time-delayed one, i.e., $0<\alpha<0.5, w_{\min }(\alpha)$ remains at a constant value of $\frac{\pi}{2}$. Note that as AD is impossible in the instantaneously coupled identical oscillators of Eq. (1) with $\alpha=0, w_{\min }(\alpha)$ has no value at $\alpha=0$. But from Fig. 4 one can state that even in coupled identical oscillators $\mathrm{AD}$ can be induced by the presence of a very small proportion of time-delayed coupling along with the instantaneous coupling rather than the completely time-delayed coupling.

In the above analysis, we have shown that for $0<\alpha<0.5$ the AD island is enclosed only by the two critical curves $\tau_{a}$ and $\tau_{b}$. The constant value of the critical threshold $w_{\min }(\alpha)=\frac{\pi}{2}$ in Fig. 4 for $0<\alpha<0.5$ can be analytically calculated from the condition of intersection of $\tau_{a}$ and $\tau_{b}$. If $w>w_{\min }(\alpha), \tau_{a}$ and $\tau_{b}$ have two different intersection points in the parameter space of $(\tau, K)$; the lower intersection point $\left(\frac{\pi}{w}, K_{\min }=\frac{1}{2 \alpha}\right)$ and the upper one $\left(\frac{\pi}{w}, K_{\max }\right)$. Unlike the case of the lower intersection point, there is no simple explicit form for $K_{\max }$, but both $K_{\min }$ and $K_{\max }$ satisfy the following equation:

$$
\cos ^{-1}\left(1-\frac{1}{\alpha K}\right)=\pi-\frac{\pi}{w} \sqrt{2 \alpha K-1} .
$$

At the critical threshold of frequency $w=w_{\min }(\alpha)$, these two intersection points collide, and the AD island vanishes. Taking the derivation of Eq. (8) with respective to $K$, we get

$$
\frac{w}{\alpha \pi}=K \text {. }
$$

By inserting $K=\frac{1}{2 \alpha}$ into Eq. (9), we have

$$
w=\frac{\pi}{2}
$$


which gives the value of the critical threshold $w_{\min }(\alpha)=\frac{\pi}{2}$ for $0<\alpha<0.5$.

The underlying mechanisms of mixed time-delayed coupling facilitating $\mathrm{AD}$ could be interpreted in the following intuitive way. It should be noticed that an $\mathrm{AD}$ state is a very special type of complete synchronization. When $\mathrm{AD}$ occurs, all coupled oscillators are attracted to the same steady state, which is unstable in the single uncoupled oscillator. The coupling between oscillators should not only make the steady state synchronous, but also convert it from an unstable to a stable one. It is well known that the presence of time delay in the coupling can successfully switch the stability of the unstable steady state, and the instantaneous coupling generally prefers complete synchronization in identical oscillators. In the proposed scheme of mixed time-delayed coupling, the time-delayed contribution tries to stabilize an AD state, while the instantaneous one favors its onset for a large set of parameters.

\section{A NETWORK OF COUPLED LIMIT-CYCLE OSCILLATORS}

Now the stabilizing effects of mixed time-delayed coupling will be demonstrated in an arbitrary network of oscillators. We employ the following network of Stuart-Landau oscillators with mixed time-delayed coupling:

$$
\begin{aligned}
\dot{z}_{j}= & \left(1+i w-\left|z_{j}\right|^{2}\right) z_{j}+\frac{(1-\alpha) K}{d_{j}} \sum_{\substack{s=1 \\
s \neq j}}^{N} g_{j s}\left(z_{s}-z_{j}\right) \\
& +\frac{\alpha K}{d_{j}} \sum_{\substack{s=1 \\
s \neq j}}^{N} g_{j s}\left[z_{s}(t-\tau)-z_{j}(t)\right]
\end{aligned}
$$

where $j=1, \ldots, N$. The network topology is characterized by the matrix $\left(g_{j s}\right)_{N \times N}$ : if nodes $j$ and $s$ are linked by an edge, then $g_{j s}=g_{s j}=1$; otherwise $g_{j s}=g_{s j}=0$. Self-links are forbidden, i.e., $g_{j j}=0 . d_{j}$ is the degree of node $j$, that is, $d_{j}=\sum_{s=1}^{N} g_{j s}$.

By performing a linear stability analysis of Eq. (11) around the AD state $z_{1}=z_{2}=\cdots=z_{N}=0$, we get a set of $N$ characteristic equations:

$$
\lambda=1+i w-K+(1-\alpha) K \rho_{j}+\alpha K \rho_{j} e^{-\lambda \tau} .
$$

Here the $\rho_{j}$ 's are the eigenvalues of the matrix $\left(\frac{g_{j s}}{d_{j}}\right)_{N \times N}$, which can be ordered as $1.0=\rho_{1} \geqslant \rho_{2} \geqslant \cdots \geqslant-\frac{1}{N-1} \geqslant$ $\rho_{N} \geqslant-1.0$. The AD island is defined by the common part of the stable regions determined by each eigenvalue $\rho_{j}$. Again, using the complex Lambert function $W[59,60]$, for each $\rho_{j}$, Eq. (12) can be solved analytically,

$$
\begin{aligned}
\lambda= & \frac{1}{\tau} W\left(\tau \alpha K \rho_{j} e^{-\left\{1+i w+\left[(1-\alpha) \rho_{j}-1\right] K\right\} \tau}\right) \\
& +1+i w+\left[(1-\alpha) \rho_{j}-1\right] K .
\end{aligned}
$$

In fact, the $\mathrm{AD}$ island is bounded by only two extreme eigenvalues $\rho_{1}$ and $\rho_{N}$, which are called the in-phase and outof-phase eigenvalues [62], respectively. Performing a similar analysis as in Sec. II, we find that the critical curves bounding the $\mathrm{AD}$ island are finally given by

$$
\begin{aligned}
\tau_{a} & =\frac{2 m \pi+\cos ^{-1}\left(1-\frac{1}{\alpha K}\right)}{w-\sqrt{2 \alpha K-1}}, \\
\tau_{b} & =\frac{2(m+1) \pi-\cos ^{-1}\left(1-\frac{1}{\alpha K}\right)}{w+\sqrt{2 \alpha K-1}}, \\
\tau_{c} & =\frac{2(m+1) \pi-\cos ^{-1}\left[\frac{K-1-(1-\alpha) K \rho_{N}}{\alpha K \rho_{N}}\right]}{w-\sqrt{\left(\alpha K \rho_{N}\right)^{2}-\left[1-K+(1-\alpha) K \rho_{N}\right]^{2}}}, \\
\tau_{d} & =\frac{2 m \pi+\cos ^{-1}\left[\frac{K-1-(1-\alpha) K \rho_{N}}{\alpha K \rho_{N}}\right]}{w+\sqrt{\left(\alpha K \rho_{N}\right)^{2}-\left[1-K+(1-\alpha) K \rho_{N}\right]^{2}}},
\end{aligned}
$$

where $m=0,2, \ldots, \infty . \tau_{a}$ and $\tau_{b}$ are derived from $\rho_{1}=1$, and $\tau_{c}$ and $\tau_{d}$ from $\rho_{N}$. If $\rho_{N}=-1$, these four critical curves are the same as in Eq. (7). If $w \leqslant 10$, then only one AD island can be found on the $(\tau, K)$ plane [26,53,54], i.e., $m=0$ in Eq. (14).

The maximal intersection point of the two curves $\tau_{c}$ and $\tau_{d}$ in Eq. (14) is deduced as $\left(\frac{\pi}{w}, \frac{1}{1+(2 \alpha-1) \rho_{N}}\right)$. The minimal intersection point of $\tau_{a}$ and $\tau_{b}$ is $\left(\frac{\pi}{w}, \frac{1}{2 \alpha}\right)$. If $\frac{1}{1+(2 \alpha-1) \rho_{N}}<\frac{1}{2 \alpha}$, i.e., $\alpha<0.5$, the $\mathrm{AD}$ island boundaries are determined only by $\tau_{a}$ and $\tau_{b}$. Thus when $0<\alpha<0.5$, the AD island expands along the axis of the coupling strength $K$ with the expansion ratio $\frac{1}{\alpha}$. To support the above analysis, we numerically study a ring network with $N=11$ nodes as an illustrative example, for which $\rho_{N}=\cos \left[\left(1-\frac{1}{N}\right) \pi\right]$. We find that on decreasing $\alpha$ from 1.0 to 0.5 the spread of AD island grows to a larger set of parameters in the $(\tau, K)$ space as shown in Figs. 5(a)-5(e)
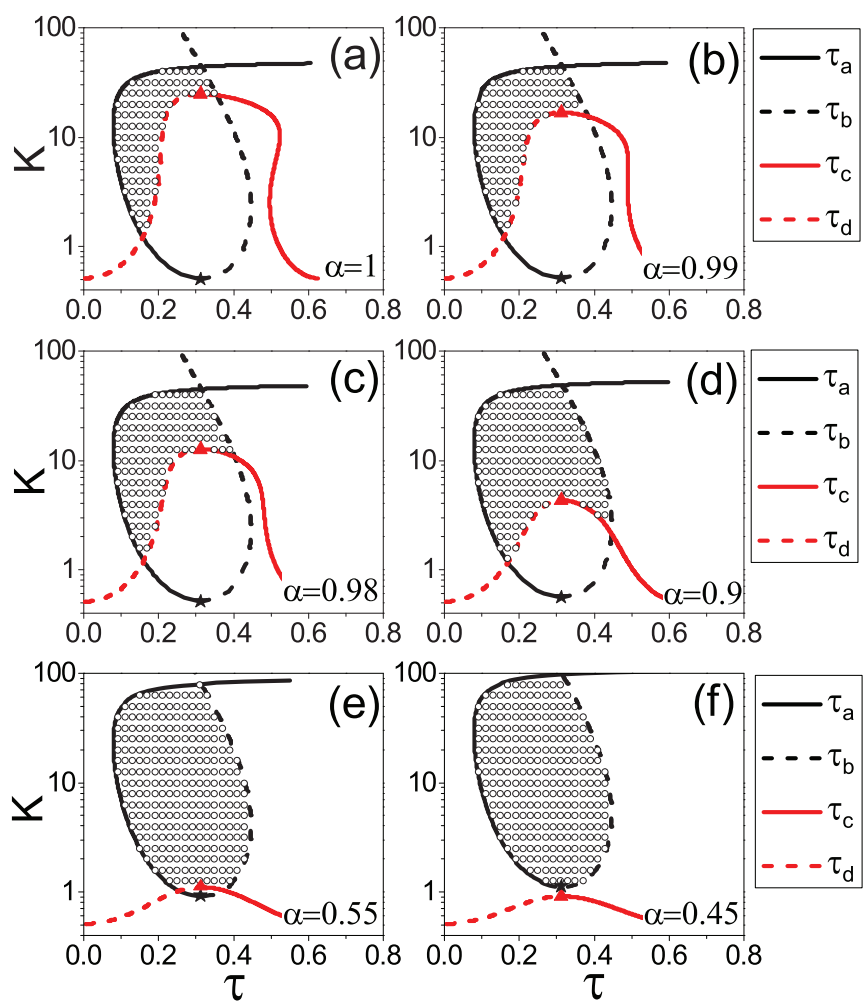

FIG. 5. (Color online) The study of the coupled systems Eq. (11) for a ring topology with $N=11 . w=10$ is fixed. The maximal point of intersection of $\tau_{c}$ and $\tau_{d}$ is given by $\left\{\pi / w, 1 /\left[1+(2 \alpha-1) \rho_{N}\right]\right\}$ marked by the red triangles. $\tau_{a}, \tau_{b}, \tau_{c}$, and $\tau_{d}$ are given in Eq. (14), which are indicated by differen colors and styles. 
for $\alpha=1,0.99,0.98,0.9$, and 0.55 , respectively. For $\alpha<0.5$, the AD island is bounded only by $\tau_{a}$ and $\tau_{b}$ as depicted in Fig. 5(f) for $\alpha=0.45$. Thus, we have observed a similar effect of mixed time-delayed coupling on networked Stuart-Landau oscillators as discussed in Sec. II for two coupled StuartLandau oscillators. We have also confirmed similar results in several other types of networks.

\section{TWO COUPLED CHAOTIC OSCILLATORS}

We have also investigated the effect of mixed time-delayed coupling on a number of other dynamical systems and find quite generally that the mixed time-delayed coupling facilitates the onset of $\mathrm{AD}$ for a large range of system parameters. Numerical results are provided here for a system of two coupled chaotic Rössler oscillators, which obeys the following equations:

$$
\begin{aligned}
\dot{x}_{1}= & w_{1}\left(-y_{1}-z_{1}\right), \\
\dot{y}_{1}= & w_{1}\left(x_{1}+a y_{1}\right)+(1-\alpha) K\left(y_{2}-y_{1}\right) \\
& +\alpha K\left[y_{2}(t-\tau)-y_{1}(t)\right], \\
\dot{z}_{1}= & w_{1}\left[b+z_{1}\left(x_{1}-c\right)\right], \\
\dot{x}_{2}= & w_{2}\left(-y_{2}-z_{2}\right), \\
\dot{y}_{2}= & w_{2}\left(x_{2}+a y_{2}\right)+(1-\alpha) K\left(y_{1}-y_{2}\right) \\
& +\alpha K\left[y_{1}(t-\tau)-y_{2}(t)\right], \\
\dot{z}_{2}= & w_{2}\left[b+z_{2}\left(x_{2}-c\right)\right],
\end{aligned}
$$

where $a=b=0.1$ and $c=14 . w_{1}>0$ and $w_{2}>0$ are the time scales of the two uncoupled Rössler oscillators. The coupled chaotic systems of Eq. (15) may be regarded as a generalization of coupled limit-cycle systems with different intrinsic frequencies, such as the two coupled Stuart-Landau oscillators in Eq. (1). In the absence of coupling ( $K=0)$, each Rössler oscillator has a phase-coherent chaotic attractor and an unstable focus $P=\left(x^{*}, y^{*}, z^{*}\right)$, where $x^{*}=-a y^{*}, y^{*}=-z^{*}$,
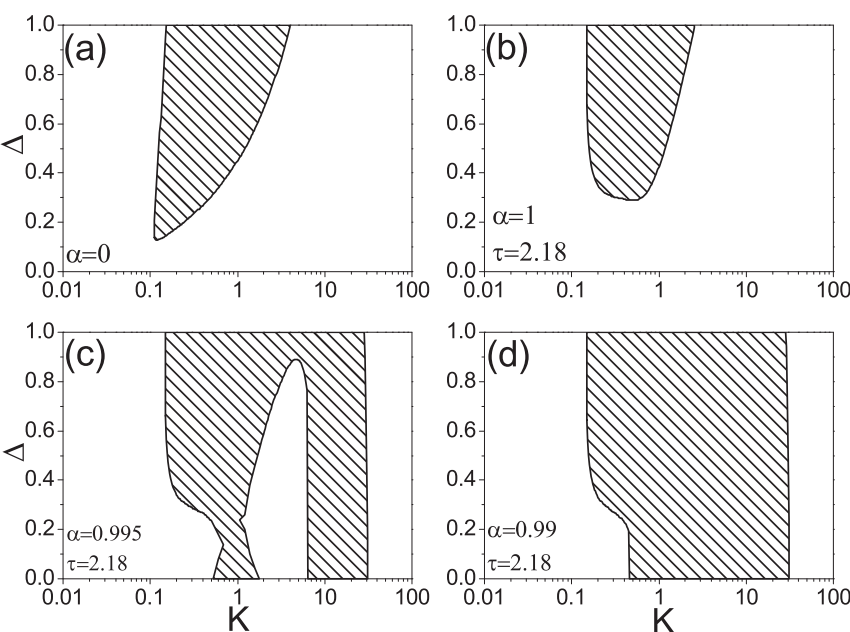

FIG. 6. The stability regions of $\mathrm{AD}$ in the two coupled nonidentical chaotic Rössler oscillators of Eq. (15) for (a) the fully instantaneous coupling with $\alpha=0$, (b) the fully time-delayed coupling with $\alpha=1.0$, and the mixed coupling with (c) $\alpha=0.995$ and (d) $\alpha=0.99$, respectively. $w_{1}=1+\Delta / 2$ and $w_{2}=1-\Delta / 2$. $\tau=2.18$ is fixed.
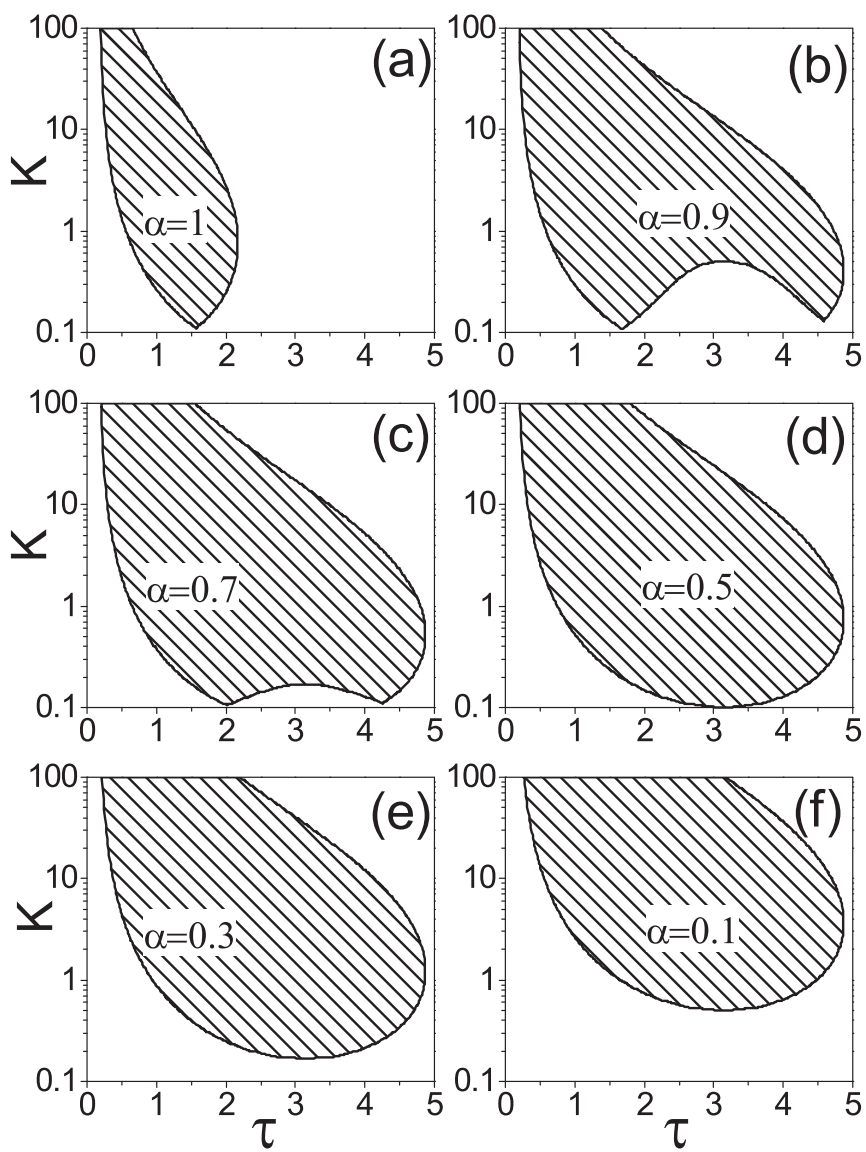

FIG. 7. The AD regions of the two coupled identical chaotic Rössler oscillators of Eq. (15) in the parameter space of $(\tau, K)$ for (a) $\alpha=1$, (b) $\alpha=0.9$, (c) $\alpha=0.7$, (d) $\alpha=0.5$, (e) $\alpha=0.3$, and (f) $\alpha=0.1$, respectively. $w_{1}=w_{2}=1$ is fixed.

and $z^{*}=\frac{c-\sqrt{c^{2}-4 a b}}{2 a}$. When $\mathrm{AD}$ takes place in the coupled systems (15), the chaotic oscillation is quenched, and all the coupled elements eventually lie on the stationary fixed point $P$.

For the coupled systems (15) to experience AD in the case of purely instantaneous coupling with $\alpha=0$, both the strength of coupling and the mismatch of two time scales should be large enough. The corresponding $\mathrm{AD}$ region in the $(K, \Delta)$ plane is displayed in Fig. 6(a). $w_{1}=1+\frac{\Delta}{2}$ and $w_{2}=1-\frac{\Delta}{2}$ are fixed. If we set the time delay at $\tau=2.18$, the $\mathrm{AD}$ region for the case of completely time-delayed coupling with $\alpha=1$ is slightly deformed as illustrated in Fig. 6(b). With the introduction of a small amount of instantaneous interaction along with the time-delayed coupling, the $\mathrm{AD}$ region expands and touches the $\Delta=0$ axis as depicted in Figs. 6(c) and 6(d) for $\alpha=0.995$ and 0.99 , respectively. The $\mathrm{AD}$ region on the $\Delta=0$ axis increases if the instantaneous contribution is enhanced by decreasing the value of $\alpha$.

Figures 7(a)-7(f) depict AD regions in the parameter space of $(\tau, K)$ for $\alpha=1,0.9,0.7,0.5,0.3$, and 0.1 , respectively, where $w_{1}=w_{2}=1$ is fixed. The $\mathrm{AD}$ region grows in size as a function of both the coupling strength and the time delay upon decreasing the value of $\alpha$ from 1 to 0.5 . Decreasing $\alpha(0<\alpha<$ $0.5)$ further, the $\mathrm{AD}$ region expands along the coupling strength $K$ direction. These observations in coupled chaotic oscillators resemble the previous results established in the coupled 
Stuart-Landau limit-cycle systems, which underscores the generality of the stabilizing effect of mixed time-delayed coupling in coupled nonlinear systems.

\section{CONCLUSION AND DISCUSSIONS}

In summary, we have examined the phenomenon of $\mathrm{AD}$ in nonlinear oscillators with mixed time-delayed coupling, which is a combination of instantaneous and time-delayed couplings in a certain proportion. Such a mixed time-delayed coupling is a more realistic and appropriate representation of the manner of interaction in real systems. We designed a parameter $\alpha$ $(0 \leqslant \alpha \leqslant 1)$ to measure the proportion of the instantaneous contribution and the time-delayed part, where the cases with $\alpha=1$ and 0 reduce to the completely time-delayed coupling and the purely instantaneous coupling, respectively. With decreasing value of $\alpha$, the time-delayed interaction in the coupling is weakened; in the meantime, the instantaneous contribution is enhanced.

We found that the mixed time-delayed coupling $(0<\alpha<$ 1) facilitates the onset of $\mathrm{AD}$ in a large set of parameter space compared to the case of purely time-delayed coupling. If the time-delayed contribution dominates the instantaneous one, i.e., $0.5 \leqslant \alpha<1$, the $\mathrm{AD}$ island grows along directions of both the time delay and the coupling strength; the minimal value of the intrinsic frequency for delay-induced AD monotonically decreases as $\alpha$ decreases. On the other hand, if the instantaneous contribution is more weighted, i.e., $0<\alpha<0.5$, the AD island expands only along the direction of coupling strength with a specific ratio $\frac{1}{\alpha}$, while the threshold value of the intrinsic frequency for delay-induced AD stays at a constant value of $\frac{\pi}{2}$. A small proportion of the time-delayed coupling along with the instantaneous coupling can give rise to $\mathrm{AD}$ in coupled identical oscillators rather than the completely time-delayed coupling. The stabilizing effect of mixed time-delayed coupling is further shown to be valid for networks of limit-cycle oscillators and for two coupled chaotic oscillators.
It is worthwhile to note that our findings are derived by performing a standard linear stability analysis. Thus, one may speculate that the results can be retrieved by starting with a system of coupled unstable foci [63-65], which ignore the nonlinear parts of the local dynamics. But the phenomenon of $\mathrm{AD}$ refers to the quenching of oscillations of the entire coupled oscillatory systems as a consequence of the interaction. Hence, the uncoupled individual systems should have oscillating behaviors rather than solely an unstable focus. In fact, random initial conditions were used to confirm the analytical predictions in the numerical experiments. Excellent agreement between analytical critical curves and numerical results was found, independent of the initial conditions, which indicates that $\mathrm{AD}$ is not only linearly stable but also globally attractive.

Chaos control is a very active topic in the research field of nonlinear science [4]. The proposed coupling scheme in this work may serve as an indicative design of additional control techniques for real-world complex systems. Studying the effects of mixed time-delayed coupling on other collective behaviors of coupled nonlinear oscillators is certainly an interesting subject for future investigations. Finally, our findings in this work should attract general interest from researchers in the fields of nonlinear dynamics and have great potential for applications in systems biology, ecology, signal processing, and neuroscience [66].

\section{ACKNOWLEDGMENTS}

We acknowledge the referees for their valuable comments which helped greatly to improve our work. This work was supported by the Alexander von Humboldt Foundation of Germany, the National Natural Science Foundation of China (Grants No. 11202082, No. 61203235, No. 11026182, No. 61104152, and No. 61175119), the Fundamental Research Funds for the Central Universities of China (Grant No. 2013QN165), the Natural Science Foundation of Jiangsu Province of China (Grant No. BK2010408), the SUMO (EU), the PHOCUS (EU), and IRTG1740 (DFG-FAPESP).
[1] A. T. Winfree, The Geometry of Biological Time (Springer, New York, 1980).

[2] Y. Kuramoto, Chemical Oscillations, Waves, and Turbulence (Springer, Berlin, 1984).

[3] A. Pikovsky, M. Rosenblum, and J. Kurths, Synchronization: A Universal Concept in Nonlinear Sciences (Cambridge University Press, Cambridge, England, 2003).

[4] Handbook of Chaos Control, edited by E. Schöll and H. G. Schuster, 2nd completely revised and enlarged ed. (Wiley-VCH, Weinheim, 2008), and references therein.

[5] M. Lakshmanan and D. V. Senthilkumar, Dynamics of Nonlinear Time-Delay Systems (Springer, Berlin, 2010).

[6] A. Arenas, A. Díaz-Guilera, J. Kurths, Y. Moreno, and C. Zhou, Phys. Rep. 469, 93 (2008).

[7] S. Guan, X. Gong, K. Li, Z. Liu, and C.-H. Lai, New J. Phys. 12, 073045 (2010).

[8] D. M. Abrams and S. H. Strogatz, Phys. Rev. Lett. 93, 174102 (2004).
[9] D. M. Abrams, R. Mirollo, S. H. Strogatz, and D. A. Wiley, Phys. Rev. Lett. 101, 084103 (2008).

[10] A. Prasad, J. Kurths, S. K. Dana, and R. Ramaswamy, Phys. Rev. E 74, 035204(R) (2006).

[11] G. Saxena, A. Prasad, and R. Ramaswamy, Phys. Rep. 521, 205 (2012).

[12] M. F. Crowley and I. R. Epstein, J. Phys. Chem. 93, 2496 (1989).

[13] K. Bar-Eli, Physica D 14, 242 (1985).

[14] M. Dolnik and I. R. Epstein, Phys. Rev. E 54, 3361 (1996).

[15] I. Ozden, S. Venkataramani, M. A. Long, B. W. Connors, and A. V. Nurmikko, Phys. Rev. Lett. 93, 158102 (2004).

[16] E. Ullner, A. Zaikin, E. I. Volkov, and J. García-Ojalvo, Phys. Rev. Lett. 99, 148103 (2007).

[17] A. Koseska, E. Volkov, and J. Kurths, Europhys. Lett. 85, 28002 (2009).

[18] A. Koseska, E. Volkov, and J. Kurths, Chaos 20, 023132 (2010).

[19] A. Prasad, Y. C. Lai, A. Gavrielides, and V. Kovanis, Phys. Lett. A 318, 71 (2003). 
[20] R. Vicente, S. Tang, J. Mulet, C. R. Mirasso, and J.-M. Liu, Phys. Rev. E 73, 047201 (2006).

[21] M. Wei and J. Lun, Appl. Phys. Lett. 91, 061121 (2007).

[22] J. Benford, H. Sze, W. Woo, R. R. Smith, and B. Harteneck, Phys. Rev. Lett. 62, 969 (1989).

[23] D. G. Aronson, G. B. Ermentrout, and N. Kopell, Physica D 41, 403 (1990).

[24] R. E. Mirollo and S. H. Strogatz, J. Stat. Phys. 60, 245 (1990).

[25] W. Liu, X. Wang, S. Guan, and C.-H. Lai, New J. Phys. 11, 093016 (2009).

[26] D. V. Ramana Reddy, A. Sen, and G. L. Johnston, Phys. Rev. Lett. 80, 5109 (1998)

[27] S. H. Strogatz, Nature (London) 394, 316 (1998).

[28] D. V. Ramana Reddy, A. Sen, and G. L. Johnston, Phys. Rev. Lett. 85, 3381 (2000).

[29] K. Konishi, Phys. Rev. E 68, 067202 (2003).

[30] K. Konishi, Int. J. Bifurcation Chaos Appl. Sci. Eng. 17, 2781 (2007).

[31] K. Konishi and N. Hara, Phys. Rev. E 83, 036204 (2011).

[32] R. Karnatak, R. Ramaswamy, and A. Prasad, Phys. Rev. E 76, 035201(R) (2007).

[33] W. Zou, X. G. Wang, Q. Zhao, and M. Zhan, Front. Phys. China 4, 97 (2009).

[34] X. M. Zhang, Y. C. Wu, and J. H. Peng, Int. J. Bifurcation Chaos Appl. Sci. Eng. 21, 225 (2011).

[35] A. Prasad, M. Dhamala, B. M. Adhikari, and R. Ramaswamy, Phys. Rev. E 81, 027201 (2010).

[36] V. Resmi, G. Ambika, and R. E. Amritkar, Phys. Rev. E 84, 046212 (2011).

[37] V. Resmi, G. Ambika, R. E. Amritkar, and G. Rangarajan, Phys. Rev. E 85, 046211 (2012).

[38] A. Sharma and M. D. Shrimali, Phys. Rev. E 85, 057204 (2012).

[39] F. M. Atay, Phys. Rev. Lett. 91, 094101 (2003).

[40] W. Zou and M. Zhan, Phys. Rev. E 80, 065204(R) (2009).

[41] G. Saxena, A. Prasad, and R. Ramaswamy, Phys. Rev. E 82, 017201 (2010).

[42] K. Konishi, H. Kokame, and N. Hara, Phys. Lett. A 374, 733 (2010).
[43] K. Konishi, H. Kokame, and N. Hara, Phys. Rev. E 81, 016201 (2010).

[44] W. Zou, D. V. Senthilkumar, Y. Tang, and J. Kurths, Phys. Rev. E 86, 036210 (2012).

[45] W. Zou, C. G. Yao, and M. Zhan, Phys. Rev. E 82, 056203 (2010).

[46] Y. N. Kyrychko, K. B. Blyuss, and E. Schöll, Eur. Phys. J. B 84, 307 (2011).

[47] W. Zou, J. Q. Lu, Y. Tang, C. J. Zhang, and J. Kurths, Phys. Rev. E 84, 066208 (2011).

[48] W. Zou, Y. Tang, L. X. Li, and J. Kurths, Phys. Rev. E 85, 046206 (2012).

[49] Y. L. Song, J. Xu, and T. H. Zhang, Chaos 21, 023111 (2011).

[50] A. Prasad, Phys. Rev. E 72, 056204 (2005).

[51] K. Konishi and K. Senda, and H. Kokame, Phys. Rev. E 78, 056216 (2008).

[52] J. M. Höfener, G. C. Sethia, and T. Gross, Phil. Trans. R. Soc. A 371, 20120462 (2013).

[53] D. V. Ramana Reddy, A. Sen, and G. L. Johnston, Physica D 129, 15 (1999).

[54] R. Dodla, A. Sen, and G. L. Johnston, Phys. Rev. E 69, 056217 (2004).

[55] K. Konishi, Phys. Rev. E 70, 066201 (2004).

[56] F. M. Atay, J. Diff. Eqns. 221, 190 (2006).

[57] W. Zou, X. Zheng, and M. Zhan, Chaos 21, 023130 (2011).

[58] B. Fiedler, V. Flunkert, P. Hövel, and E. Schöll, Philos. Trans. R. Soc., A 368, 319 (2010).

[59] J. K. Hale, Functional Differential Equations (Springer, New York, 1971).

[60] A. Amann, E. Schöll, and W. Just, Physica A 373, 191 (2007).

[61] Y. Song, J. Wei, and Y. Yuan, J. Nonlinear Sci. 17, 145 (2007).

[62] M. P. Mehta and A. Sen, Phys. Lett. A 355, 202 (2006).

[63] A. G. Balanov, N. B. Janson, and E. Schöll, Phys. Rev. E 71, 016222 (2005).

[64] S. Yanchuk, M. Wolfrum, P. Hövel, and E. Schöll, Phys. Rev. E 74, 026201 (2006)

[65] T. Dahms, P. Hövel, and E. Schöll, Phys. Rev. E 76, 056201 (2007).

[66] Y. Tang, H. Gao, W. Zou, and J. Kurths, PLoS ONE 7, 41375 (2012). 